Bull. Mater. Sci., Vol. 21, No. 3, June 1998, pp. 219-226. (C) Indian Academy of Sciences.

\title{
Dielectric properties and electrical conduction in yttrium iron garnet (YIG)
}

\author{
LALITHA SIRDESHMUKH*, K KRISHNA KUMAR, S BAL LAXMAN, A RAMA KRISHNA \\ and G SATHAIAH \\ Department of Physics, Kakatiya University, Warangal 506009 , India
}

MS received 28 April 1997; revised 5 March 1998

\begin{abstract}
The dielectric properties (dielectric constant and loss) of a single crystal of yttrium iron garnet $\left(\mathrm{Y}_{3} \mathrm{Fe}_{5} \mathrm{O}_{12}\right)$ were measured in the temperature range 77-725 $\mathrm{K}$ and in the frequency range $100 \mathrm{~Hz}-1 \mathrm{MHz}$. AC conductivity was derived from dielectric constant and loss. DC conductivity was measured in the temperature range $30-725 \mathrm{~K}$. Thermoelectric power (TEP) was measured from $77-800 \mathrm{~K}$. On the basis of the results, conduction in this garnet is interpreted as due to small polarons. The nature of conduction at different temperature ranges is discussed in the light of existing reports on defect formation.
\end{abstract}

Keywords. Yttrium iron garnet; dielectric constant; conductivity; polaron conduction.

\section{Introduction}

Yttrium iron garnet (YIG) exhibits ferrimagnetism with low hysteresis loss and high resistivity at room temperature. Because of this, it is of technical importance and finds use in radio-electronics and UHF technology (Krupichka 1976). Hence the study of magnetic and electrical properties is important and useful.

The magnetic properties of YIG have been investigated by many workers (Epstein and Frackiewicz 1959; Wurlitzer and Richter 1970; Wurlitzer 1987; Bush 1988; Chukalkin et al 1989). There are some reports on studies on electrical conductivity (Elwell and Dixon 1968; Fontana and Epstein 1971; Metselaar and Larsen 1974; Lal et al 1982; Petrov et al 1986). There are only a few reports on dielectric properties. Hirakata et al (1991) reported the dielectric behaviour at liquid nitrogen temperatures but the dielectric constant was not clearly indicated. Hofmeister and Campbell (1992) determined low- and high-frequency dielectric constants from infrared reflectivity measurements and indicated that the data by direct measurement is not available for a pure YIG single crystal.

From resistivity and thermoelectric power measurements on $\mathrm{Hf}^{+}$-doped $\mathrm{YIG}$ in the range $500-1000 \mathrm{~K}$, Elwell and Dixon (1968) concluded that the conduction should be described by localized model rather than the band model. Studies on electrical conduction in silicondoped YIG by Fontana and Epstein (1971) in the temperature range $20-200^{\circ} \mathrm{C}$ is interpreted as arising from localized hopping of electron amongst four $\mathrm{Fe}^{3+}$ cation sites around $\mathrm{Si}^{4+}$ impurity sites.

*Author for correspondence
From DC conductivity and thermoelectric power measurements, Metselaar and Larsen (1974) indicate the possibility of $p$-type conduction in YIG in the high temperature range $1200-1700 \mathrm{~K}$. Results of Lal et al (1982) and Petrov et al (1986) in high temperature range $>600 \mathrm{~K}$ and $>1270 \mathrm{~K}$, respectively support conduction via thermally-activated hopping of $p$-type charge carriers.

As evident from literature survey, while dielectric properties have been measured only in the liquid nitrogen range of temperature, resistivity measurements have been done on a $\mathrm{Hf}^{4+}$-doped sample at higher temperature beyond $500 \mathrm{~K}$. Electrical conductivity studies have been made up to $475 \mathrm{~K}$ on a polycrystalline sample. The authors have neither come across a detailed investigation of dielectric properties over wide range of temperatures and frequencies nor conductivity measurements on a single crystal sample.

It is well-known that the measurement of dielectric loss is a powerful method to detect relaxation due to formation of dipoles. $\mathrm{AC}$ conductivity in a wide range of frequencies combined with $\mathrm{DC}$ conductivity and thermoelectric power measurements helps to understand the conduction mechanism and identify the charge carriers responsible for conduction.

Based on the above-mentioned facts, the measurements of dielectric constant and loss, DC conductivity and thermoelectric power have been carried out on a single crystal of YIG. The results are discussed to understand the conduction mechanism.

\section{Experimental}

All the measurements were carried out on a flux-grown 
single crystal of YIG with parallel (111) faces. The sample was well polished and was in the form of a semi-circular disk of dimensions $0.67 \mathrm{~cm}^{2} \times 0.0615 \mathrm{~cm}$.

In the low-temperature region, capacitance and loss were recorded using a fully automated computercontrolled CGA-83 transformer ratio arm bridge. The bridge was capable of measurement in three-terminal configuration. The accuracy of capacitance and loss is $0.1 \%$ and $1 \%$, respectively. The measurements were carried out at 17 frequencies in the range $10-100 \mathrm{kHz}$ at temperatures from $77 \mathrm{~K}$ to $340 \mathrm{~K}$ with an interval of $5 \mathrm{~K}$ and with an accuracy of $0.1 \mathrm{~K}$.

The high-temperature dielectric measurements were carried out using a Hewlett-Packard $4192 \mathrm{~A}$ impedance analyzer in conjunction with a laboratory-designed cell, in two-terminal method. The sample was coated with silver paint to ensure good electrical contact. Lowtemperature resistivity measurements were carried out using an automated temperature controller and a Keithley digital electrometer. A Keithley electrometer (model $610 \mathrm{C}$ ) was used for high-temperature DC conductivity measurements with an applied voltage of 3 volts.

For low-temperature resistivity and thermoelectric power measurements, laboratory-built sample holders were used with a closed cycle helium cryo-refrigerator as the cooling device. The temperature gradient was controlled by using a Lakeshore temperature controller with an accuracy in temperature of $0.1 \mathrm{~K}$. Hightemperature thermoelectric power measurements were carried out using differential method and a Keithley nano-voltmeter for recording the data.

\section{Results}

\subsection{Dielectric properties and conductivity}

3.1a Low-temperature measurements: The low-temperature data was recorded as capacitance $C(\omega)$ and $G(\omega), \omega$ as a function of temperature, where $G(\omega)$ is

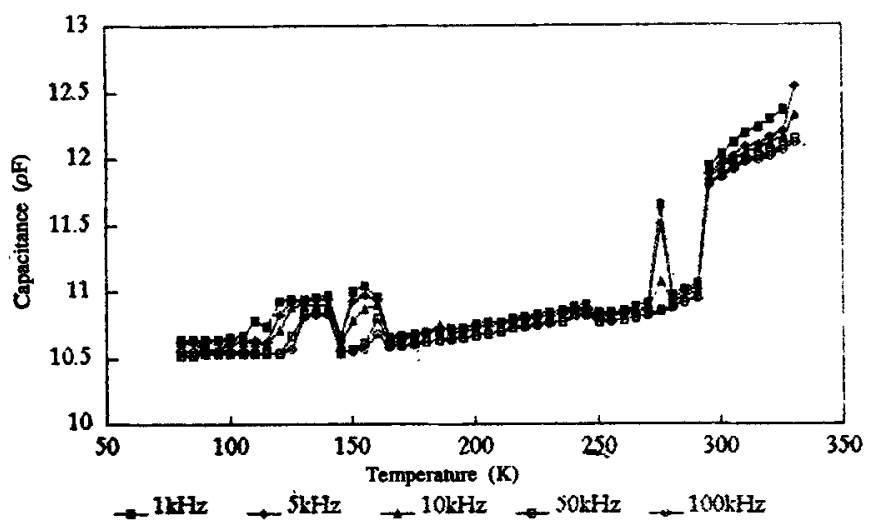

Figure 1. Variation of capacitance with temperature at different frequencies. conductance. The variations are to be treated as relative variations in dielectric constant and loss. The temperature variation of capacitance and loss at a few selected frequencies are shown in figures 1 and 2 , respectively. The change in capacitance with frequency from $1-100 \mathrm{kHz}$ is only $1 \%$ in low-temperature range and $2 \%$ around $340 \mathrm{~K}$. The variation with temperature is slow with distinct peaks around $130 \mathrm{~K}, 160 \mathrm{~K}$ and a sharp increase at $280 \mathrm{~K}$. This increase may be attributed to a large increase in ionic contribution to dielectric constant. Hirakata et al (1991) observed some anomalies in the real part of the dielectric constant and loss around 130 $\mathrm{K}$. They believe that these changes reflect the changes observed in the first-order magnetoelectric effect experiments of Kita et al (1988). Kita et al (1988) attributed the changes to some sort of phase transition, leading to a non-centrosymmetric structure below $125 \mathrm{~K}$.

Such a transformation could not be detected by X-ray and Mössbauer studies (Hirakata et al 1991). According to Hirakata et al (1991), the lattice strain due to the structural transition is so small that it could not be detected by X-ray, electron diffraction, Mössbauer and dielectric measurements. However, they support the existence of some sort of phase transformation, on the basis of the fact that the second-order magnetoelectric effect also shows a sharp decrease around $125 \mathrm{~K}$ (Takano et al 1991).

The variation of loss $(G / \omega)$ with temperature is shown in figure 2. The peaks around $130 \mathrm{~K}, 160 \mathrm{~K}$ and $285 \mathrm{~K}$ are the manifestation of the effects observed in magnetic dispersion curves due to the presence of $\mathrm{Si}^{4+}$ in YIG (Wurlitzer and Richter 1970). Other studies on YIG such as magnetoelectric effect (Hirakata et al 1991) mentioned earlier and studies on magnetic effect by Torres $e t$ al (1993) indicate peaks around $130 \mathrm{~K}$ and $180 \mathrm{~K}$. The effect is believed to be due to the electron transfer between $\mathrm{Fe}^{2+}$ and $\mathrm{Fe}^{3+}$ ions creating local order and induced anisotropy. The temperature at which large increase is observed i.e. $295 \mathrm{~K}$ corresponds to $\theta / 2$ where

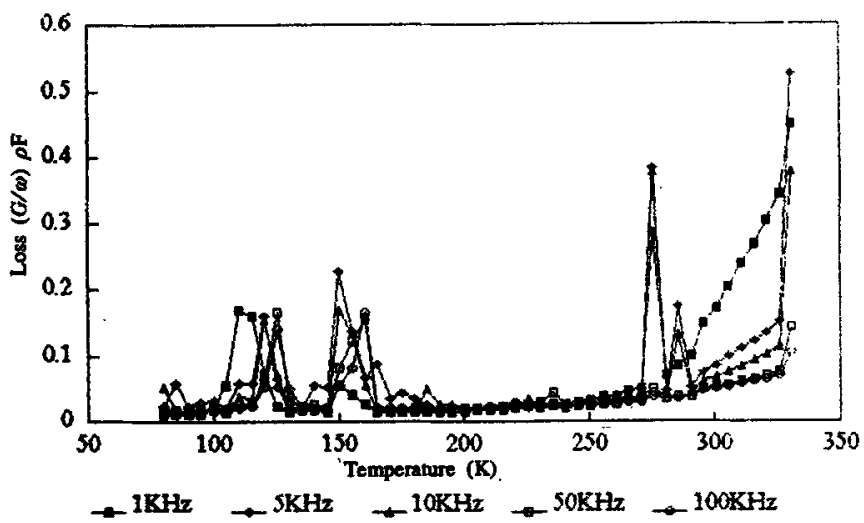

Figure 2. Variation of loss $(G / \omega)$ with temperature at different frequencies. 
$\theta(600 \mathrm{~K})$ is Debye temperature (Landolt-Bornstein 1991). AC conductivity $\sigma_{\mathrm{AC}}$ is obtained from the data on dielectric constant $(\varepsilon)$ and loss, $\tan \delta$ using the relation

$$
\sigma_{\mathrm{AC}}=\varepsilon \varepsilon_{0} \omega \tan \delta,
$$

where $\varepsilon_{0}$ is the vacuum permittivity and $\omega$ the angular frequency. Figure 3 shows plots of $\mathrm{AC}$ conductivity against reciprocal temperature. For clarity, the regions of peaks in dielectric loss are not shown. It can be seen that conductivity is frequency-dependent and temperature-independent up to $250 \mathrm{~K}$. The low-temperature DC conductivity (figure 4) is found to be independent of temperature. (Relative variation with temperature is to be noted and not the absolute value).

In the magnetic loss studies in YIG, Wurlitzer and Richter (1970) and Wurlitzer (1987) observed magnetic loss dispersion when a small amount of silicon is present as impurity which gets incorporated during growth or as dopant. This is attributed to the electron hopping between $\mathrm{Fe}^{3+}$ and $\mathrm{Fe}^{2+}$ ions. The crystal used for the present measurements is grown by flux in which divalent impurities $\mathrm{Pb}, \mathrm{Ca}, \mathrm{Fe}^{2+}$ and $\mathrm{F}$ are present in addition to $\mathrm{Si}^{4+}$ (Haussuhl 1976).

3.1b High temperature measurements: Initially, the dielectric constant and loss were measured as functions

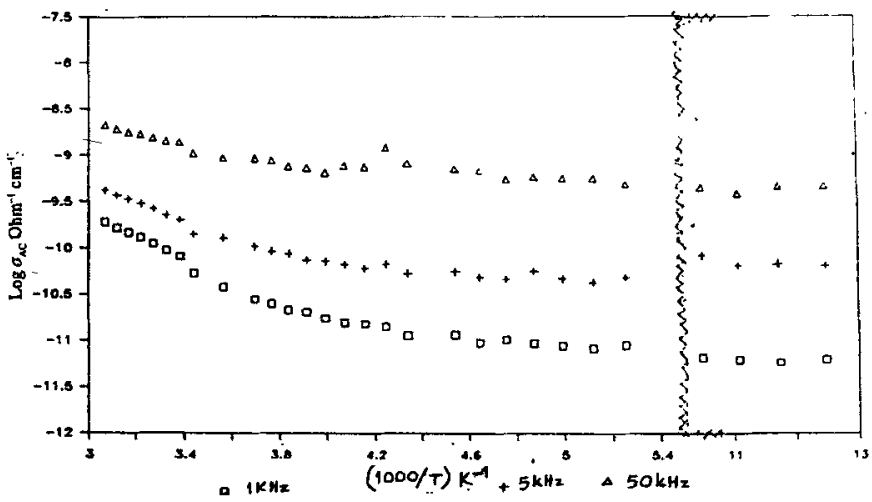

Figure 3. Variation of $\mathrm{AC}$ conductivity with reciprocal temperature at different frequencies. of frequency at room temperature. It is well established that for ionic crystals an increase in $\varepsilon$ and loss $(\tan \delta)$ at low frequencies is observed for samples due to voids, dislocations and other defects. These spurious effects cease to influence the value beyond a certain frequency. The frequency-independent value is taken as static dielectric constant. For the sample of YIG used for measurements, the dielectric constant is almost frequencyindependent beyond $50 \mathrm{kHz}$ at room temperature. Values of the dielectric constant obtained in the present work and those reported by earlier workers are given in table 1. The values obtained from direct measurement are close to one another.

The variation of dielectric constant with temperature at different frequencies is shown in figure 5. It can be seen that large variations with temperature are observed at low frequencies. The variation of loss with temperature at different frequencies is shown in figure 6 . The curves show well-defined peaks. The peaks show a shift to higher frequencies for higher temperatures indicating that they are relaxation peaks. The activation energy for the relaxation process is obtained from the plot of log peak frequency $\left(\omega_{\mathrm{p}}\right)$ against reciprocal temperature shown in figure 7 . The activation energy is calculated to be $1.01 \mathrm{eV}$.

The temperature variation of AC conductivity is shown in the form of a $\log \sigma$ vs $T^{-1}$ plot (figure 8). It can be

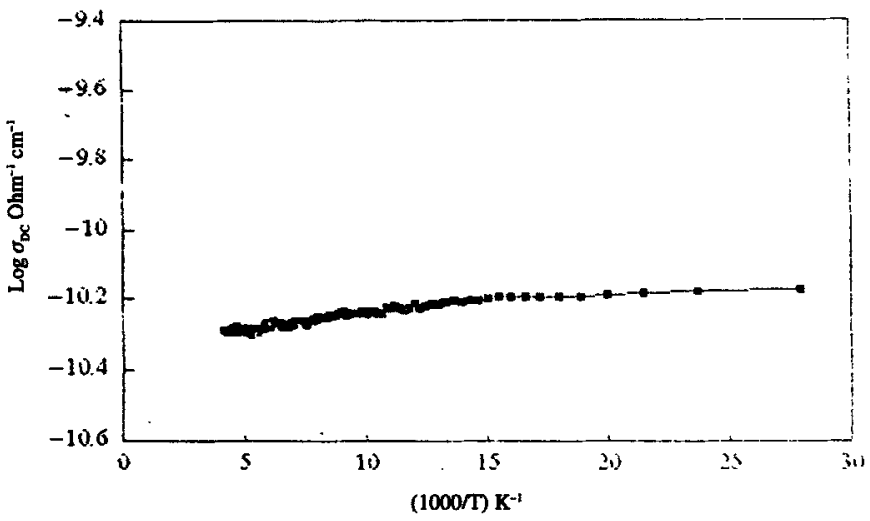

Figure 4. Variation of DC conductivity with reciprocal temperature.

Table 1. Dielectric constant of YIG.

\begin{tabular}{|c|c|c|c|}
\hline Sample & $\begin{array}{l}\text { Frequency } \\
\text { (temp.) }\end{array}$ & $\begin{array}{l}\text { Dielectric } \\
\text { constant }\end{array}$ & Reference \\
\hline Polycrystalline & $2 \mathrm{MHz}$ (R.T.) & $\sim 12$ & Anderson (1959) \\
\hline Single crystal & $\begin{array}{l}\text { Spectroscopic } \\
\text { analysis (R.T.) }\end{array}$ & $19 \cdot 4$ & Hofmeister and Campbell (1992) \\
\hline Single crystal & $1 \mathrm{MHz}$ (R.T.) & $12 \cdot 5$ & Present work \\
\hline
\end{tabular}


seen that a frequency-dependent slow increase is observed up to $500 \mathrm{~K}$. The conductivity is found to be high for higher frequencies as in the case of variation at low temperatures discussed earlier. At $500 \mathrm{~K}$, all the curves merge at a point showing frequency independence. The frequency dependence is shown once again beyond $500 \mathrm{~K}$ which is rather an unusual behaviour. These measurements were repeated to check for consistency. The same type of variation was observed in repeated measurements. The increase with temperature is faster beyond $500 \mathrm{~K}$ with higher values at lower frequencies and beyond $670 \mathrm{~K}$ an abrupt increase of more than two orders is observed.

The DC conductivity in the temperature range from room temperature to $700 \mathrm{~K}$ is shown in figure 9 . Room temperature conductivity is of the order of $10^{-13} \mathrm{mho}$ $\mathrm{cm}^{-1}$. It reaches an order of $10^{-4} \mathrm{mho} \mathrm{cm}^{-1}$ around $670 \mathrm{~K}$ and still higher beyond that temperature. It is to be noted that distinct kinks are observed at $500 \mathrm{~K}$ and $575 \mathrm{~K}$. The temperature at which the first kink is observed corresponds to the point at which a frequency-independent conductivity is observed in AC conductivity just at one fixed temperature. Perhaps the nature of conduction

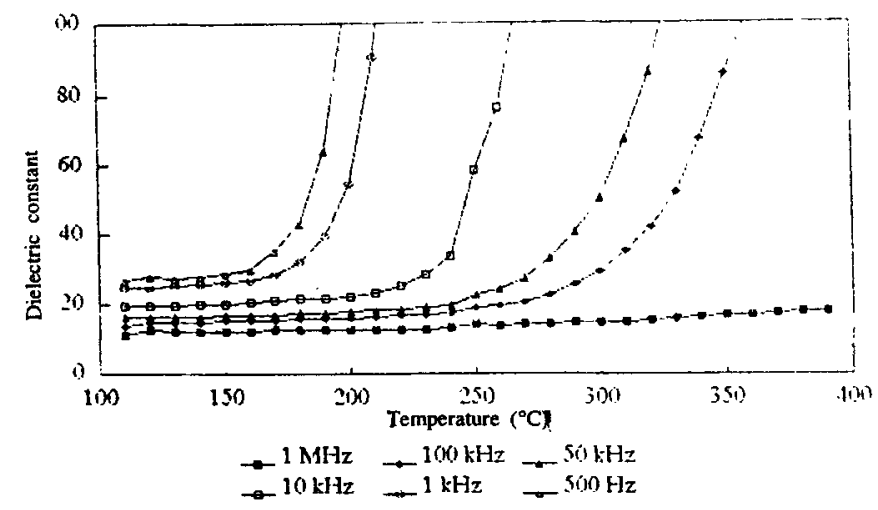

Figure 5. Variation of dielectric constant with temperature at different frequencies.

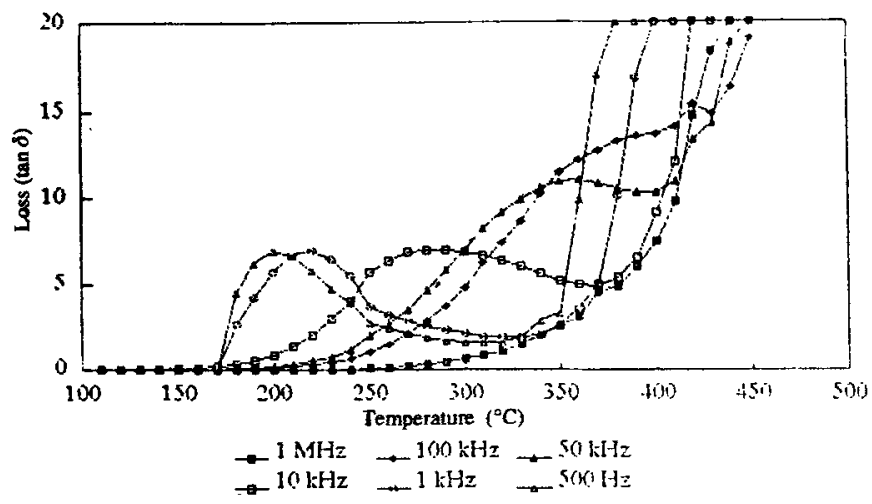

Figure 6. Variation of $\tan \delta$ with temperature at different frequencies. changes beyond this point as discussed earlier. The second kink at higher temperature corresponds to Neel temperature of YIG $(573 \mathrm{~K})$. Lal et al (1982) observed similar variation for several iron garnets at the Neel temperature. For a related material, DyIG, Yadav and Lal (1979) have reported a break in the $\log \sigma$ vs $T^{-1}$ curve at magnetic ordering temperature. Change in the activation energy for conduction near curie temperature has been observed for Ti-substituted YIG by Om Prakash and Bahadur (1982).

The significance of such an observation can not be explained at this stage. It can only be said that magnetic ordering has some effect on conductivity. In the entire region, conduction is extrinsic and $\mathrm{AC}$ conductivity is higher than DC conductivity.

\subsection{Thermoelectric power}

The variations of thermoelectric power with temperature in the low- and high-temperature ranges are shown in figures $10 \mathrm{a}$ and $\mathrm{b}$, respectively. The thermoelectric voltage, $\Delta E$ developed across the sample over a

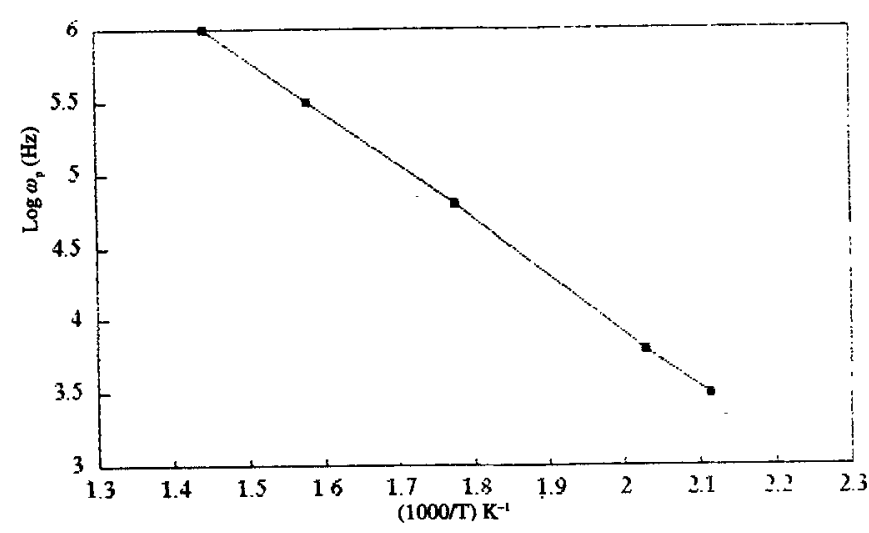

Figure 7. Variation of $\log \omega_{\mathrm{p}}$ with reciprocal temperature.

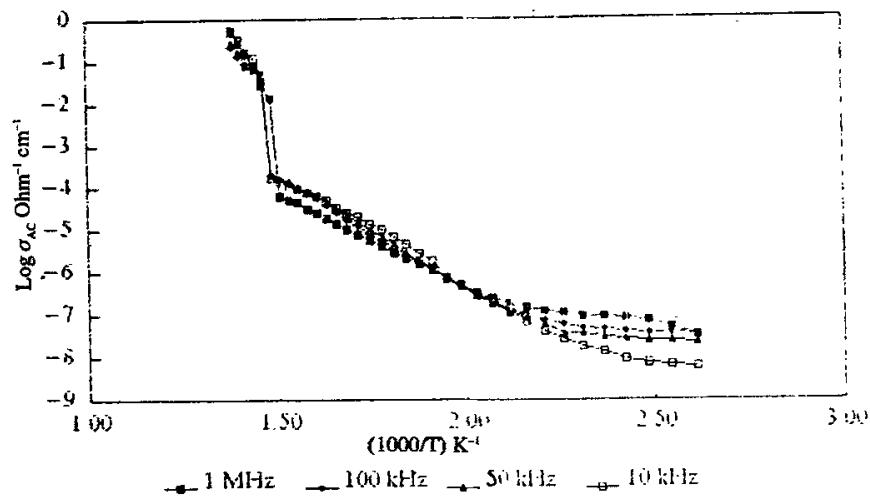

Figure 8. Variation of $\mathrm{AC}$ conductivity with reciprocal temperature at different frequencies. 
temperature gradient, $\Delta T$ is shown as $S=\Delta E / \Delta T$. Following the convention adopted by Lal et al (1982), the positive sign of $S$ indicates negative charge carriers and vice versa. In the low-temperature range the thermoelectric power shows a gradual increase while decrease is observed beyond room temperature up to $700 \mathrm{~K}$. Similar variation with an increase to a certain temperature has been observed in other oxide compounds (Om Prakash et al 1994). The charge carriers are $n$-type as shown by the sign of the thermoelectric power. The value remains almost constant at higher temperatures beyond $700 \mathrm{~K}$.

\section{Discussion}

YIG shows $n$-type conduction due to the presence of substitutional cations or anion vacancies. Doping of YIG with $\mathrm{Si}^{4+}$ induces creation of $\mathrm{Fe}^{2+}$ ions. The simultaneous presence of $\mathrm{Fe}^{3+}$ ions alter the electric and magnetic properties of garnets. The anisotropic magnetic resonance and slow relaxation phenomenon are examples of the electron exchange between the two irons (Judy 1966; Wurlitzer 1987). These effects in magnetic properties have been extensively studied as mentioned earlier. The sample used for the present investigation has marginal excess of donor concentrations. The sample characterization is given as $\mathrm{Si}^{4+}(0.003)+\mathrm{F}(0.02)$ (Haussuhl 1976) against the sum of divalent impurities $\mathrm{Fe}^{2+}$ $(0.01)+\mathrm{Ca}^{2+}(0.001)+\mathrm{Pb}(0.011)$ (concentrations are expressed in the units of ions per formula unit of YIG). The presence of anion vacancies also introduces additional amounts of donor concentrations.

YIG is a cubic crystal $\left(\mathrm{I}_{\mathrm{a}} 3_{\mathrm{d}}-\mathrm{O}_{\mathrm{h}}^{10}\right)$ with a structural chemical formula $\mathrm{Y}_{3} \mathrm{Fe}_{3}^{3+} \mathrm{Fe}_{2}^{3+} \mathrm{O}_{12}^{2-}$. The trivalent iron cations

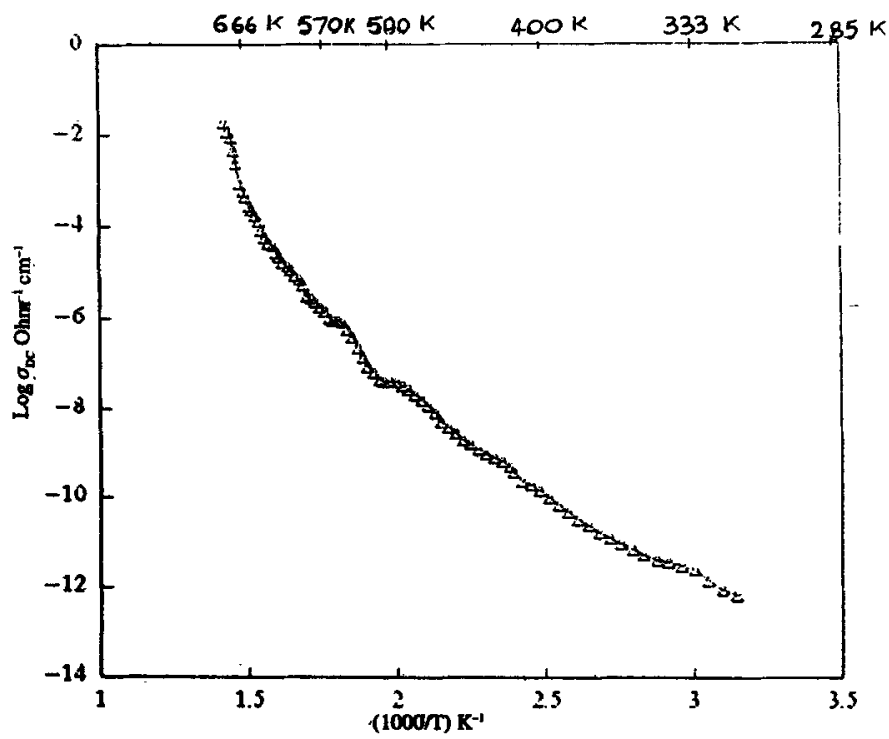

Figure 9. Variation of DC conductivity with reciprocal temperature. are distributed over two different sets of lattice sites; ' $d$ ' sublattice in which each cation is tetrahedrally surrounded by oxygen ions and ' $a$ ' sublattice with octahedral coordination. The yttrium ions occupy ' $c$ ' sites characterized by a dodecahedral oxygen coordination. The $\mathrm{Si}^{4+}$ ions are known to structurally enter the ' $d$ ' sites forcing the reduction of some of the octahedrally-coordinated $\mathrm{Fe}^{3+}$ ions to $\mathrm{Fe}^{2+}$. The $\mathrm{Fe}^{2+}$ ion may be considered as $\mathrm{Fe}^{3+}$ cation plus an electron. The dipole formed with this electron and the positive charge located at tetrahedral 'd' sites lead complex polarizability. This accounts for the relaxation phenomenon observed in dielectric loss. As the temperature is increased, some of the electrons get ionized and participate in electrical conduction. The dipolar component gives an additional term in $\mathrm{AC}$ field. The AC conductivity is found to be two orders more than the DC conductivity. Thus conduction is due to the localized charge carriers. Metselaar and Larsen (1974) have proposed a model for the band structure for YIG. They are of the opinion that in dealing with the band
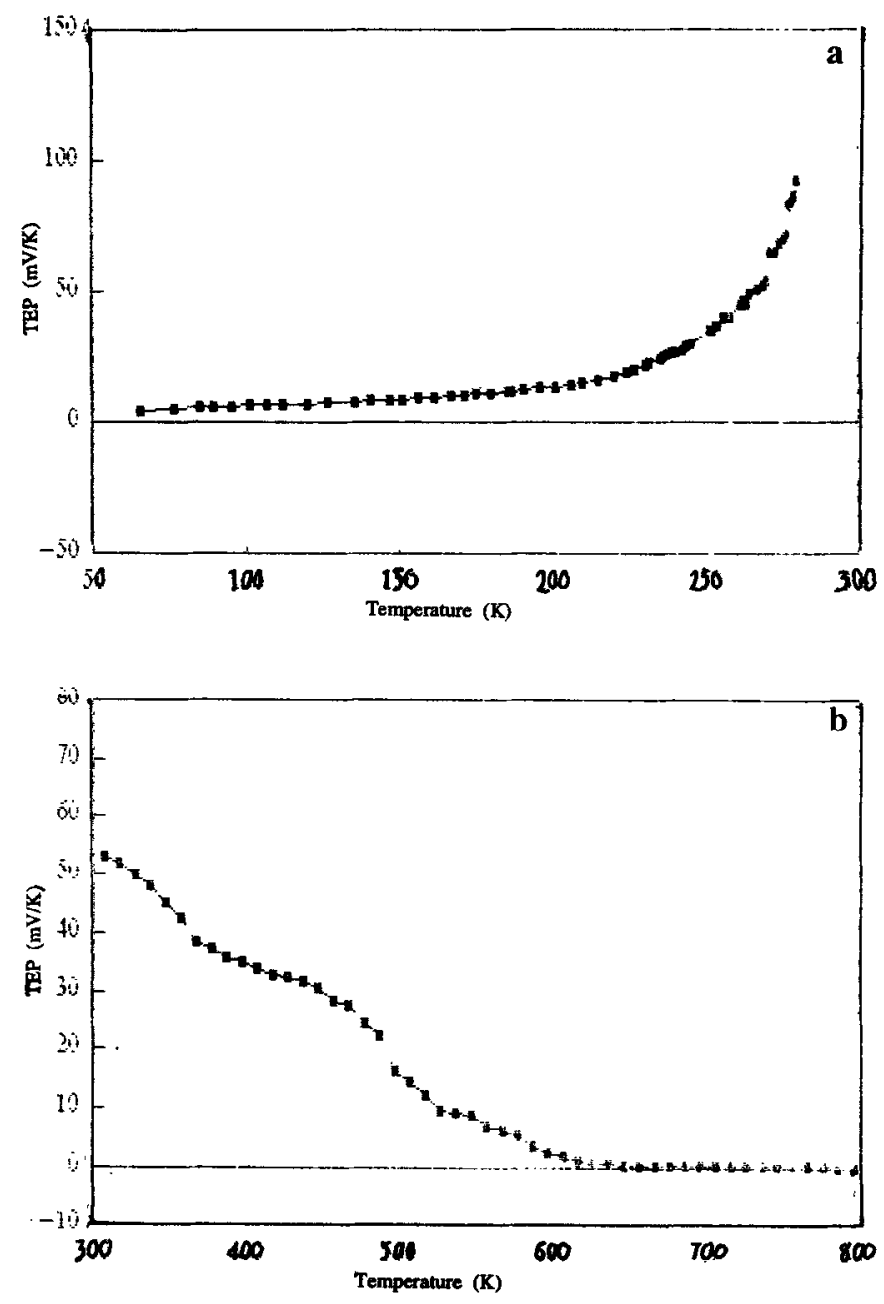

Figure 10. a-b. Variation of thermoelectric power with temperature. 
structure of YIG the problems are similar to the case of transition metal oxides like NiO. Hence the conduction takes place in the narrow band derived from $\mathrm{Fe}(3 d)$ orbitals which are octahedral in character. Lal et al (1982) proposed energy band diagram for rare earth garnets. $\mathrm{Fe}^{3+}-3 d$ bands are shown to lie above the top of the uppermost completely filled $\mathrm{O}^{2-}-2 p$ band.

Under normal situation these garnets should exhibit high metallic conduction. Experimentally it is found that pure garnets are insulators with conductivity less than $10^{-12}$ at room temperature. It may be concluded that $\mathrm{Fe}^{3+}-3 d$ bands are localized. Hence in the presence of $\mathrm{Si}^{4+}$ impurity the mode of conduction could only be due to localized electrons. The conduction mechanism can be understood by treating it as narrow band semiconductor as in the case of transition metal oxides and rare earth compounds.

Investigations of electrical conductivity in transition metal oxides (Adler and Feinleib 1970), rare earth sesquioxides (Subba Rao et al 1970; Lal et al 1976; Lal and Dar 1976) and in rare earth perovskites (Gschneid-

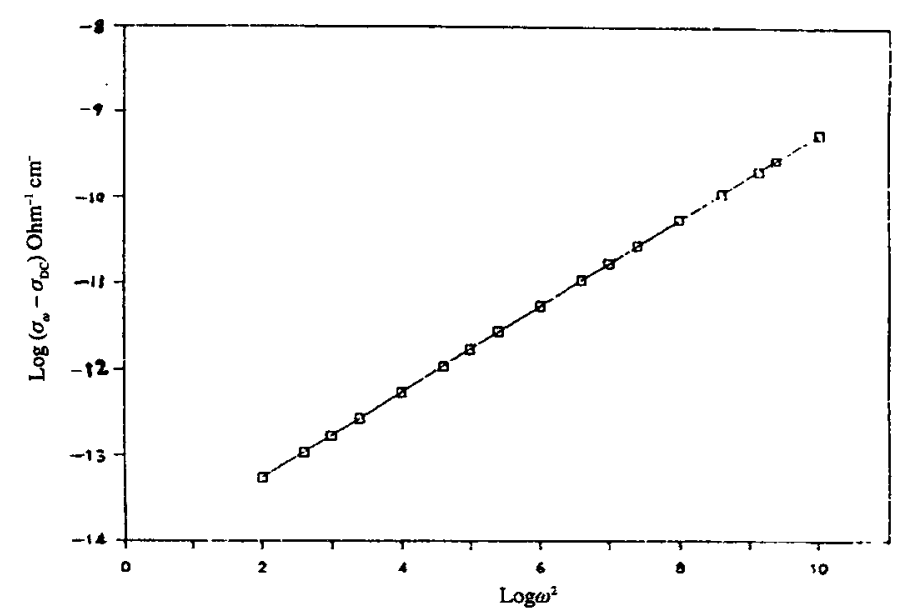

Figure 11. Variation of $\log \left(\sigma_{\omega}-\sigma_{\mathrm{DC}}\right)$ with $\log \omega^{2}$ at $90 \mathrm{~K}$. ner and Eyring 1979) indicated polaron conduction through hopping mechanism. Though for DyIG conduction, thermally-activated hopping mechanism has been proposed by Yadav and Lal (1979). Om Prakash and Bahadur (1982) have explained the electrical properties of Ti-substituted YIG on the basis of electron hopping model as suggested by Austin and Mott (1969). For YIG, small polaron hopping mechanism is proposed by Petrov et al (1986) at high temperatures.

Theoretical work by several workers over the years has provided some understanding of conduction in oxides and transition metal compounds (Bosman and Van Daal 1970). For these materials, the interaction between electrons and optical phonons is strong and the conduction is explained on the basis of polarons.

The treatment of conduction by polarons is discussed by several workers (Appel 1968; Austin and Mott 1969; Mott and Davis 1971). Polarons belong to two categories, large and small polarons. In the large polaron model, the conductivity is by band mechanism at all temperatures and the $\mathrm{AC}$ conductivity decreases with frequency. The small polarons conduct in band-like manner up to a certain temperature, the conductivity showing an increase

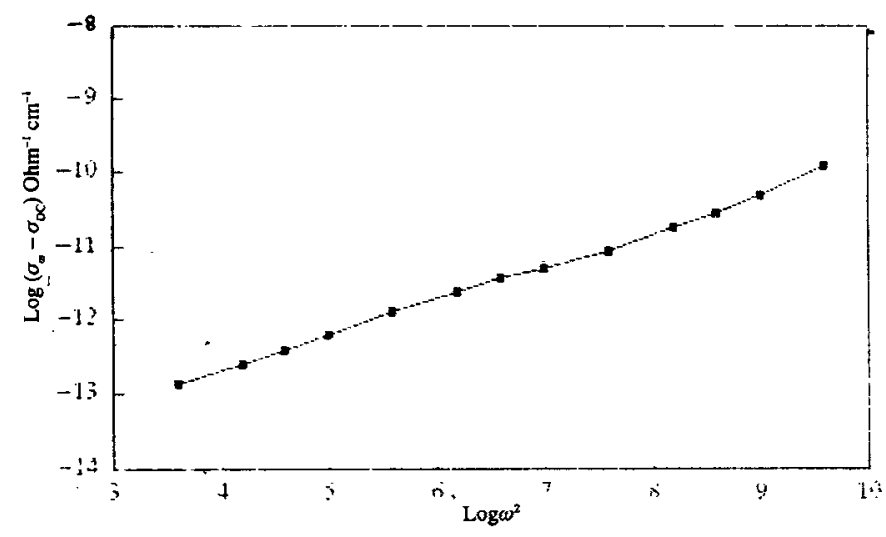

Figure 12. Variation of $\log \left(\sigma_{\omega}-\sigma_{\mathrm{DC}}\right)$ with $\log \omega^{2}$ at $200 \mathrm{~K}$

Table 2. Polaron parameters.

\begin{tabular}{|c|c|c|}
\hline Crystal parameters & Polaron parameters & Calculated values \\
\hline 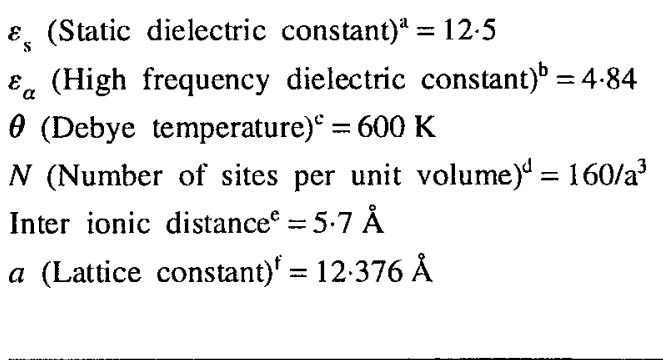 & $\begin{array}{l}r_{\mathrm{p}}=1 / 2[\pi / 6 \mathrm{~N}]^{1 / 3} \\
\omega_{0}=2 \pi K \theta / h \\
\varepsilon_{\mathrm{p}}=\varepsilon_{\mathrm{s}} \varepsilon_{\alpha} /\left[\varepsilon_{\mathrm{s}}-\varepsilon_{\alpha}\right] \\
W_{\mathrm{p}}=e^{2} /\left[8 \pi \varepsilon_{0} \varepsilon_{\mathrm{p}} r_{\mathrm{p}}\right] \\
m^{*}=20 \varepsilon_{\mathrm{p}}^{2} h^{2} \omega_{\mathrm{p}} / e^{2} \\
\alpha=e^{2}\left[m^{*} / 2 h^{3} \omega_{\mathrm{e}}\right]^{1 / 2} / \varepsilon_{\mathrm{p}} \\
m_{\mathrm{p}}=0.02 m^{*} \alpha^{4}\end{array}$ & $\begin{array}{l}0.918 \AA \\
7.85 \times 10^{13} \mathrm{~Hz} \\
7.900 \\
0.993 \mathrm{eV} \\
5.88 \times 10^{2} \mathrm{~m}_{\mathrm{e}} \\
13.85 \\
4.33 \times 10^{5} \mathrm{~m}_{\mathrm{c}}\end{array}$ \\
\hline
\end{tabular}

${ }^{a}$ Present value, ${ }^{b}$ Hofmeister and Campbell (1992). ${ }^{c}$ Landolt-Bornstein (1991), ${ }^{d}$ Geller (1960), ${ }^{\mathrm{e}}$ Austin and Mott (1969) and ${ }^{\mathrm{f}}$ Gschneidner and Eyring (1979). 
with frequency. At higher temperatures, the conduction is by thermally-activated hopping mechanism. The experimental results in the present case also shows the trend expected for small polaron conduction mechanism. It has been shown (Appel 1968; Adler 1968) that for ionic solids the concept of small polaron conduction is valid.

As an evidence for small polaron conduction, we have examined the variation of conductivity with frequency on the basis of relaxation process, as applied to bound small polaron (Adler and Feinleib 1970; Bosman and Van Daal 1970). Adler and Feinleib (1970) have shown that for conduction by small polarons the conduction increases with frequency and the following relation holds good:

$$
\sigma_{\omega}-\sigma_{\mathrm{DC}}=\frac{\omega^{2} \tau^{2}}{1+\omega^{2} \tau^{2}}
$$

where $\omega$ is the angular frequency and $\tau$ the staying time $\left(\sim 10^{-10} \mathrm{sec}\right)$ for frequencies $\omega^{2} \tau^{2}<1 . \log \left(\sigma_{t \omega}-\sigma_{\mathrm{DC}}\right)$ vs $\log \omega^{2}$ should be a straight line. Plots of $\log \left(\sigma_{\omega}-\sigma_{\mathrm{DC}}\right)$ as a function of $\omega^{2}$ at two temperatures $90 \mathrm{~K}$ and $200 \mathrm{~K}$, are shown in figures 11 and 12 , respectively. It can be seen that the plots are straight lines. This indicates that the conduction is due to small polarons.

The important parameters associated with polarons are: polaron radius, $r_{\mathrm{p}}$, polaron binding energy, $W_{\mathrm{p}}$, effective mass, $m_{\mathrm{p}}$ and dimensionless electron-phonon coupling constant $\alpha$. The polaron parameters were evaluated from the data on dielectric constant and other crystal parameters using the relations given by Austin and Mott (1969). The input data and the polaron parameters are shown in table 2.

For small polarons $r_{\mathrm{p}}$ should be less than the inter ionic distance and the coupling constant should be greater than 6 (Bosman and Van Daal 1970). The values of the parameters are appropriate for small polarons.

\section{Conclusions}

The static dielectric constant for YIG is found to be 12.5 at room temperature at $1 \mathrm{MHz}$ frequency. The disturbances observed in the dielectric constant around $130 \mathrm{~K}, 160 \mathrm{~K}$ and in the dielectric loss at three temperatures $130 \mathrm{~K}, 160 \mathrm{~K}$ and $285 \mathrm{~K}$ correspond to the peaks observed in magnetic loss and induced anisotropy measurements on silicon-doped samples. No indication of phase transition is observed. From $\mathrm{AC}$ and DC conductivity measurements, creation of $\mathrm{Fe}^{2+}$ due to the presence of $\mathrm{Si}^{4+}$ as impurity and electron hopping between $\mathrm{Fe}^{2+}$ and $\mathrm{Fe}^{3+}$ ions is believed to be the cause for conduction. Thermoelectric power data shows $n$-type charge carriers up to $700 \mathrm{~K}$.

The conduction is proposed to be due to the formation of small polarons involving band-like mechanism at low temperatures and by thermally-activated hopping mechanism at high temperatures. The relaxation pattern observed in the dielectric loss and the frequency variation of conductivity at low temperatures support this conclusion.

\section{Acknowledgements}

We thank the referee for critical scrutiny of the paper and for many valuable suggestions. The authors also thank Prof. S Haussuhl, University of Koln, and Prof. W Tolkdorf, Germany, for the gift of garnet crystal. The authors are grateful to the Director, IUC; Dr A Ganesan, Indore, and Prof. S V Suryanarayana and Dr P Venugopal Reddy, Osmania University, Hyderabad for providing laboratory facilities. The senior author (LSD) is grateful to Prof. Donald E Scheule and Dr Z Z Zhong, Case Western Reserve University, USA, for providing facilities for the low-temperature dielectric measurements. Financial assistance from the Department of Science and Technology, New Delhi, is gratefully acknowledged.

\section{References}

Adler D 1968 Solid state physics (eds) F Seitz, D Turnbull and H Ehrenreich (NY: Academic Press) 21 p. 1

Adler D and Feinleib 1970 Phys. Rev. B2 3112

Appel J 1968 Solid state physics (eds) F Seitz, D Turnbull and

H Ehrenreich (NY: Academic Press) 21 p. 193

Anderson E E 1959 J. Appl. Phys. Suppl. 30299 S

Austin I G and Mott N F 1969 Adv. Phys. 1841

Bosman A J and Van Daal H J 1970 Adv. Phys. 191

Bush G G 1988 J. Appl. Phys. 645653

Chukalkin Y G, Shtirz V R and Goshchitskii B N 1989 Phys. Status Solidi (a) 112161

Elwell D and Dixon A 1968 Solid State Commun. 6585

Epstein D J and Frackiewicz 1959 J. Appl. Phys. Suppl. 30295

Fontana Jr. R E and Epstein D J 1971 Met. Res. Bull. 6959

Geller S $1960 \mathrm{~J}$. Appl. Phys. Suppl. 3130

Gschneidner Jr. K A and Eyring L 1979 Hand book of physics and chemistry of rare earths 3 (New York: North-Holland and Physics Publishing Co)

Haussuhl S 1976 Naturforsch Z a31 390

Hirakata S, Tanaka M, Kohn K, Kita E, Siratori E, Kimura S and Tasaki A 1991 J. Phys. Soc. Jap. 60294

Holstein T 1959 Ann. Phys. 8343

Hofmeister A M and Campbell K R 1992 J. Appl. Phys. 72 638

Judy J H 1996 J. Appl. Phys. 371328

Kita E, Takano S, Tasaki A, Siratori K, Kohn K and Kimura S 1988 J. Appl. Phys. 645659

Krupichka S 1976 The physics of ferrites and magnetic oxides related to them (Russian Translation) (Moscow: Mir) 1st Edn

Lal H B, Verma B K and Dar N 1976 Indian J. Cryogenics 1119

Lal H B and Dar N 1976 Indian J. Pure \& Appl. Phys. 14 788

Lal H B, Verma B K and Ram Yadav V 1982 J. Mater. Sci. 173317 
Landolt-Bomstein 1991 Numerical Data and Functional Relationships in Science and Technology (New York: Springer Verlag) 27

Metselaar R and Larsen P K 1974 Solid State Commun. 15291

Mott N F and Davis E A 1971 Phonons and polarons in electronics processing in non crystalline materials (Oxford: Clarendon Press)

Om Prakash and Bahadur D 1982 J. Appl. Phys. 538228

Om Prakash, Devendra Kumar and Durga Prasad Ch 1994 J. Phys. D: Appl. Phys. 271509

Petrov A N, Denisov G V and Zhukovskii U M 1986 Inorg. Mater. (USA) 22579
Subba Rao G V, Ramdas S, Mehrotra P N and Rao C N R 1970 J. Solid State Chem. 2377

Takano S, Kita E, Siratori K, Kohn K, Kimura S and Tasaki A 1991 J. Phys. Soc. Jap. 60288

Torres $\mathbf{L}$, de Francisco D, Munoz $\mathrm{J} \mathbf{M}$, Zazo $\mathrm{M}$ and Iniguez J I $1993 \mathrm{~J}$. Appl. Phys. 736301

Wurlitzer M 1987 Phys. Status Solidi (a) 100607

Wurlitzer $M$ and Richter 1970 Phys. Status Solidi (a) 3 K211

Yadav V R and Lal H B 1979 Jap. J. Appl. Phys. 18 2229 